\title{
Fremiab
}

\section{Modeling Heavy Ion Ionization Energy Loss at Low and Intermediate Energies*}

\author{
I.L. Rakhno \\ Fermilab, P.O. Box 500, Batavia, Illinois 60510
}

November 17, 2009

\begin{abstract}
The needs of contemporary accelerator and space projects led to significant efforts made to include description of heavy ion interactions with matter in general-purpose Monte Carlo codes. This paper deals with an updated model of heavy ion ionization energy loss developed previously for the MARS code. The model agrees well with experimental data for various projectiles and targets including super-heavy ions in low- $Z$ media.
\end{abstract}

\section{Introduction}

The MARS code is developed for detailed Monte Carlo modeling of hadronic and electromagnetic cascades in realistic geometry for various accelerator, shielding, detector and space applications. The recent needs of the Relativistic Heavy-Ion Collider, Facility for Rare Isotope Beams, Large Hadron Collider and NASA projects served as an incentive to implement heavy-ion collision and transport physics into the MARS15 code [1]. The present paper describes the following updates to our previously developed model of ionization energy loss [2]: (i) a modified Thomas-Fermi expression for ion effective charge, $Z_{\text {eff }}$, based on that by Pierce and Blann [3]; (ii) taking into account available information on probabilities of different ion charge states for few-electron heavy ions at intermediate energies [4]. When developing the modified expression for ion effective charge, our purpose was both keeping the simplicity of the expression by Pierce and Blann and taking into account the experimentally observed dependence of $Z_{\text {eff }}$ on target material [5]. Comparisons with experimental data are presented as well.

${ }^{*}$ Work supported by Fermi Research Alliance, LLC, under contract DE-AC02-07CH11359 with the U. S. Department of Energy. 


\section{Updated Model of Ionization Energy Loss}

Ionization loss of a heavy ion is usually represented as a product of proton ionization loss and the ion effective charge, $Z_{e f f}$. Such an approach is based on results of various authors who studied correlations between experimental data on proton and heavy ion stopping power (see, e.g., [3], [5] and [6] and references therein). For super-heavy ions, however, validity of the approach is still questionable.

At energies above $1 \mathrm{MeV} /$ nucleon, ion effective charge is usually described with an expression either by Pierce and Blann [3] or by Hubert et al. [5], while at lower energies the formalism by J. Ziegler et al. is preferable [7]. Recently another dataset was introduced [8] that describes effective charges from $1 \mathrm{keV} /$ nucleon up to $1000 \mathrm{MeV} /$ nucleon for ions from ${ }_{3} \mathrm{Li}$ up to ${ }_{18} \mathrm{Ar}$. In our model, three energy regions are used in order to describe the heavy ion effective charge: (i) at energies below $1 \mathrm{MeV} /$ nucleon the formalism by J. Ziegler [7] is used; (ii) we modified the expression by Pierce and Blann [3] in order to take into account dependence of the effective charge on target material, and the modified expression is used at energies above $3 \mathrm{MeV} /$ nucleon; (iii) an interpolation is performed for energies between 1 and $3 \mathrm{MeV} /$ nucleon. As an extra option, at energies above $100 \mathrm{MeV} /$ nucleon a distribution of ion charge states can be used instead of the effective charge and such a replacement leads to a better description of experimental data on stopping power of heavy ions at energies 100-300 MeV/nucleon [4].

Having defined the ion effective charge that serves, in particular, as a multiplicative factor to be applied to proton stopping power, one uses the following algorithm in order to describe a heavy ion stopping power:

1. At energies below $10 \mathrm{MeV} /$ nucleon, tabular data on proton stopping power from [9] and [7] is used.

2. At energies between 30 and $80 \mathrm{MeV} /$ nucleon, a weighted average between two components is used. The first component is proton stopping power according to the Bethe-Bloch formalism with appropriate corrections calculated for the proton. The second component is the same proton stopping power according to the Bethe-Bloch formalism but with the corrections calculated for the ion using $Z_{e f f}$.

3. At enetgies between 10 and $30 \mathrm{MeV} /$ nucleon, an interpolation between 1.) and 2.) is performed.

4. At energies above $80 \mathrm{MeV} /$ nucleon, proton stopping power is calculated according to the Bethe-Bloch formalism with appropriate corrections calculated for the ion using $Z_{\text {eff }}$.

The corrections mentioned above are calculated according to [10]-[11] and include the following: shell, Barkas, and Lindhard-Sørensen corrections. The latter describes corrections to the first Born approximation which the Bethe-Bloch theory is based on. 


\subsection{Modified expression for ion effective charge}

The expression for the ion effective charge from [3] was obtained using experimental data for $5-90-\mathrm{MeV}$ beams of ${ }^{32} \mathrm{~S},{ }^{35} \mathrm{Cl},{ }^{79} \mathrm{Br}$, and ${ }^{127} \mathrm{I}$ as well as data for similar ions published by other authors, so that ions heavier than ${ }^{127}$ I were not taken into account. The expression did not include any dependence on target material:

$$
\frac{Z_{e f f}}{Z_{1}}=1-\exp \left(-0.95 v_{r}\right),
$$

where the reduced velocity $v_{r}$ is $v /\left(v_{0} Z_{1}^{2 / 3}\right), Z_{1}$ is projectile atomic number, $v$ is projectile velocity, and $v_{0}=e^{2} / \hbar$.

At the same time, experimental data made available approximately two decades later clearly demonstrated some dependence of the ion effective charge on target material, and the effect is the most pronounced for super-heavy ions like uranium [5]. In order to fit the experimental data, the authors introduced an expression with six parameters and did include a dependence on target material. From a numerical standpoint, however, that number of parameters can be considered as a drawback because of a possibility to get the same value of the fitting function for various combinations of parameters. The latter can give rise to an irregular behavior of the predicted effective charge $v s$ projectile or target atomic number. This statement may be confirmed by the fact that the authors initially provided a set of the six fitted parameters for nine target materials, from $\mathrm{Be}$ up to $\mathrm{Au}$, but later they reduced the amount of such target materials to two (Be and $\mathrm{C}$ ) and introduced some smoothing in their fitting procedure [12].

We made an attempt to keep the simplicity of the expression (1) and introduce a dependence on target material atomic number, $Z_{2}$, in it, so that we consider the following expression:

$$
\frac{Z_{e f f}}{Z_{1}}=1-\exp \left(-0.95 v_{r} f_{c}\right),
$$

where the correction function, $f_{c}\left(Z_{2}\right)$, for projectiles with $Z_{1}$ lower than 35 is assumed to be equal to 1 . We performed a fitting using the latter expression and experimental data collected in [5] and [12] for various ions $\left(\mathrm{O}, \mathrm{Ar}, \mathrm{Kr},{ }^{132} \mathrm{Xe},{ }^{208} \mathrm{~Pb},{ }^{238} \mathrm{U}\right)$ and for a number of target materials. The result of the fitting is presented in Fig. 1. Such a dependence reproduces the experimentally observed reduction of the effective charge with target atomic number [5]. Comparisons with experimental data on stopping power for various ions are given in Sec. 3.1.

\subsection{Distributions of few-electron ion charge states}

Using an ion effective charge in ionization loss theory at non-relativistic energies represents, in particular, an approximation when a realistic ion charge-state distribution is replaced with an averaged ion charge. At present, detailed information on such distributions 
for various projectile-target combinations in a wide energy range hardly exists. At ion energies of a few hundred $\mathrm{MeV} /$ nucleon, however, the ions are not fully stripped and keep only a few electrons. For this energy range there are several computer codes, based on pre-calculated atomic interactions cross sections, that allow us to predict such charge-state distributions [13]. Using the information, ionization energy loss of a heavy ion can be presented in the following form:

$$
\frac{d E}{d x}=\sum_{i} p_{i} \frac{d E}{d x}\left(q_{i}\right),
$$

where $p_{i}$ is a probability of finding the ion in charge state $q_{i}$ and $\frac{d E}{d x}\left(q_{i}\right)$ is corresponding stopping power. We implemented the possibility of using charge-state distributions into the MARS code and for this purpose the distributions provided with the code CHARGE were used. Detailed comparisons with experimental data on stopping powers in this energy region are given in $\mathrm{Sec}$. 3.2.

\section{Comparison with Experimental Data}

\subsection{Low energy region}

First of all, we present a comparison between experimental data and our model for the case of super-heavy ions in low- $Z$ media that demonstrates the accuracy of description of ion effective charge. In Fig. 2 one can see that in these cases the sophisticated formalism from [5] has no advantage over our model.

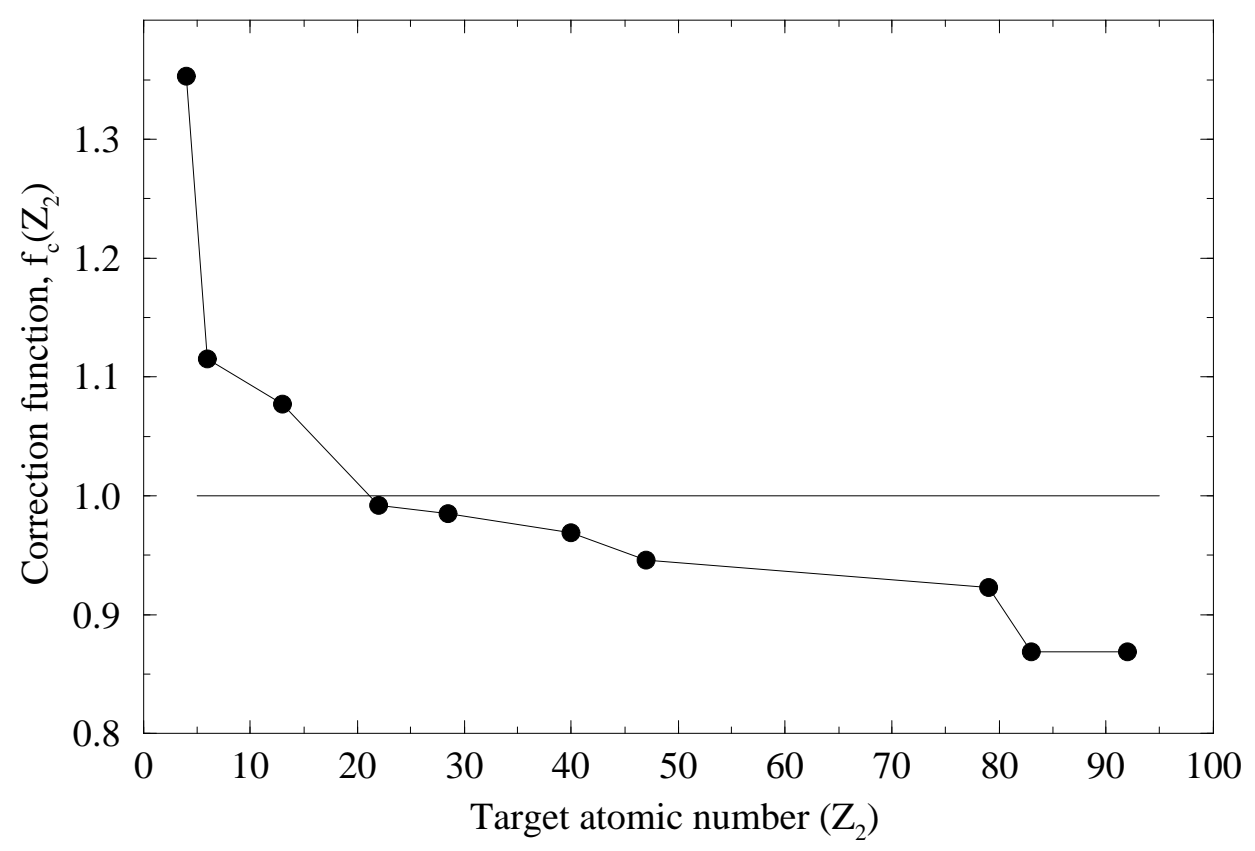

Figure 1: Calculated correction function for the expression (2). 

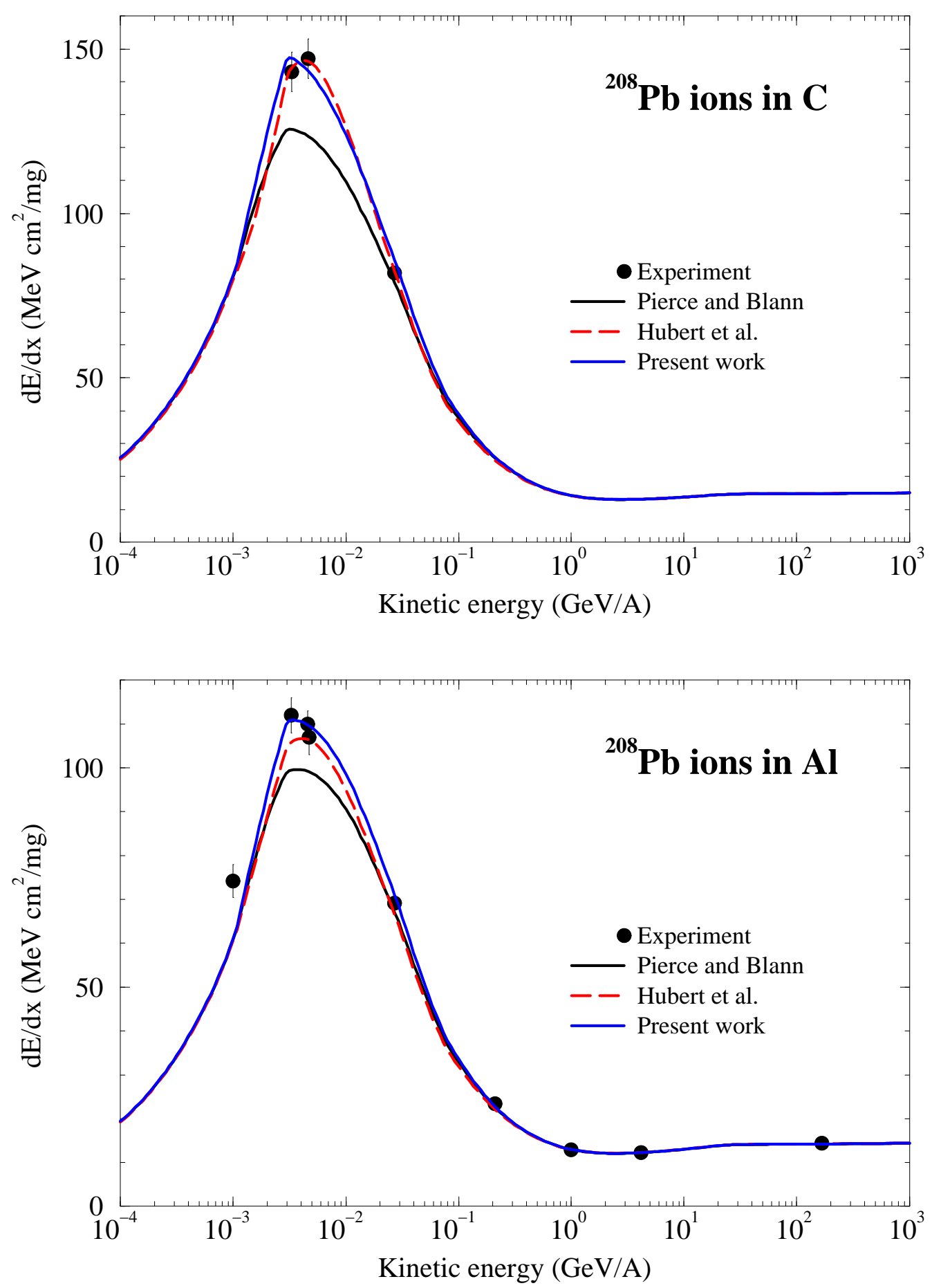

Figure 2: Comparisons between experimental data [5] on stopping power of ${ }^{208} \mathrm{~Pb}$ ions and the model predictions for the case of different expressions for ion effective charge.

Comparisons between experimental data on ion stopping powers and our model for a number of heavy ions and for light, medium and heavy target nuclei at energies from a fraction of MeV/nucleon up to $100 \mathrm{MeV} /$ nucleon are given in Figs. 3 thru 5. 
The overall agreement is good. Noticeable discrepancies are observed only at energies 1 $\mathrm{MeV} /$ nucleon and below. In order to partially resolve the problem at such low energies and down to $1 \mathrm{keV} /$ nucleon, one plans to replace the effective charge description from [7] with the formalism from [8].
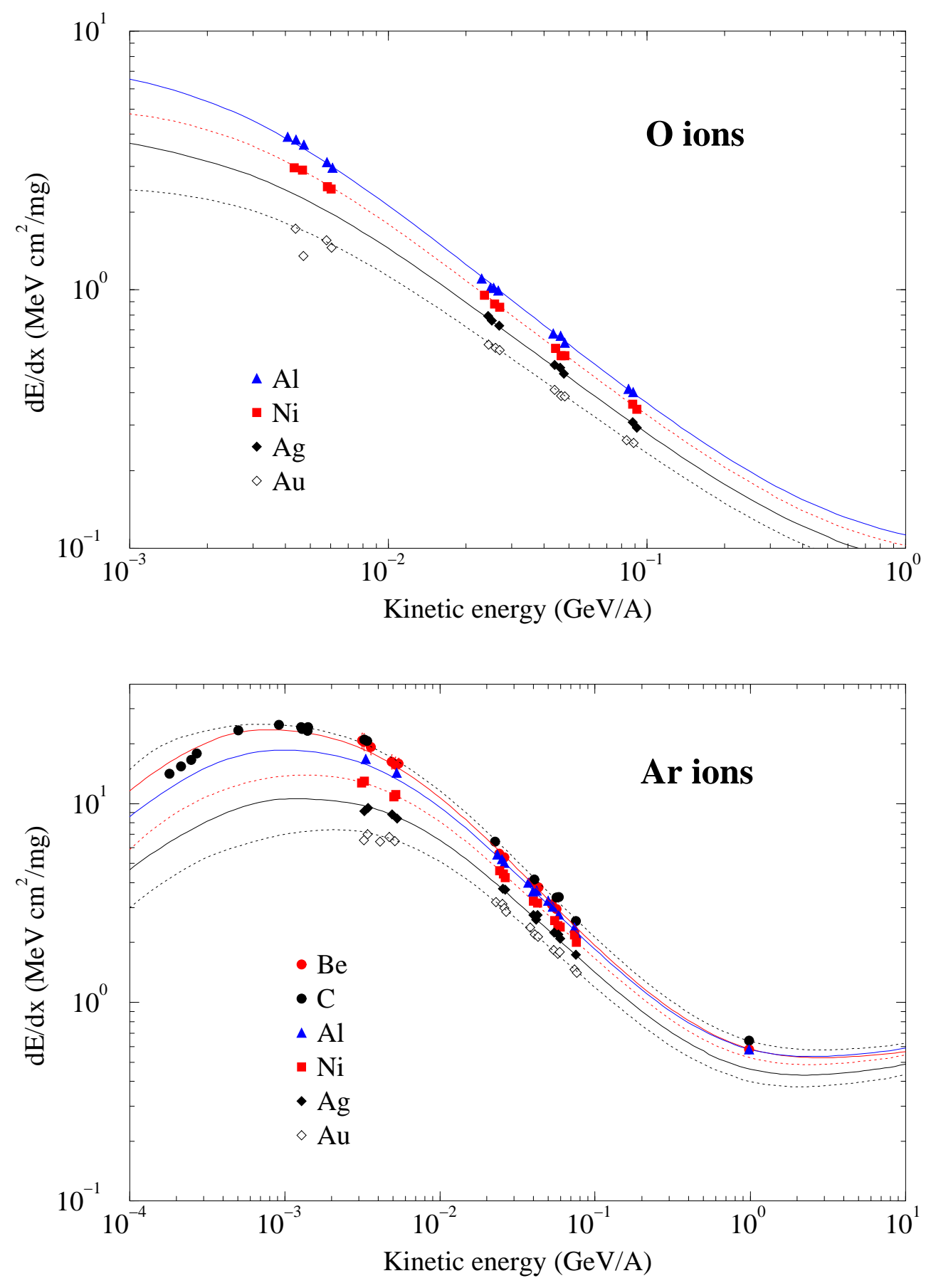

Figure 3: Comparisons between experimental data [5] (symbols) and our model (lines) on stopping power of ${ }^{16} \mathrm{O}$ and ${ }^{40} \mathrm{Ar}$ ions in various targets. 

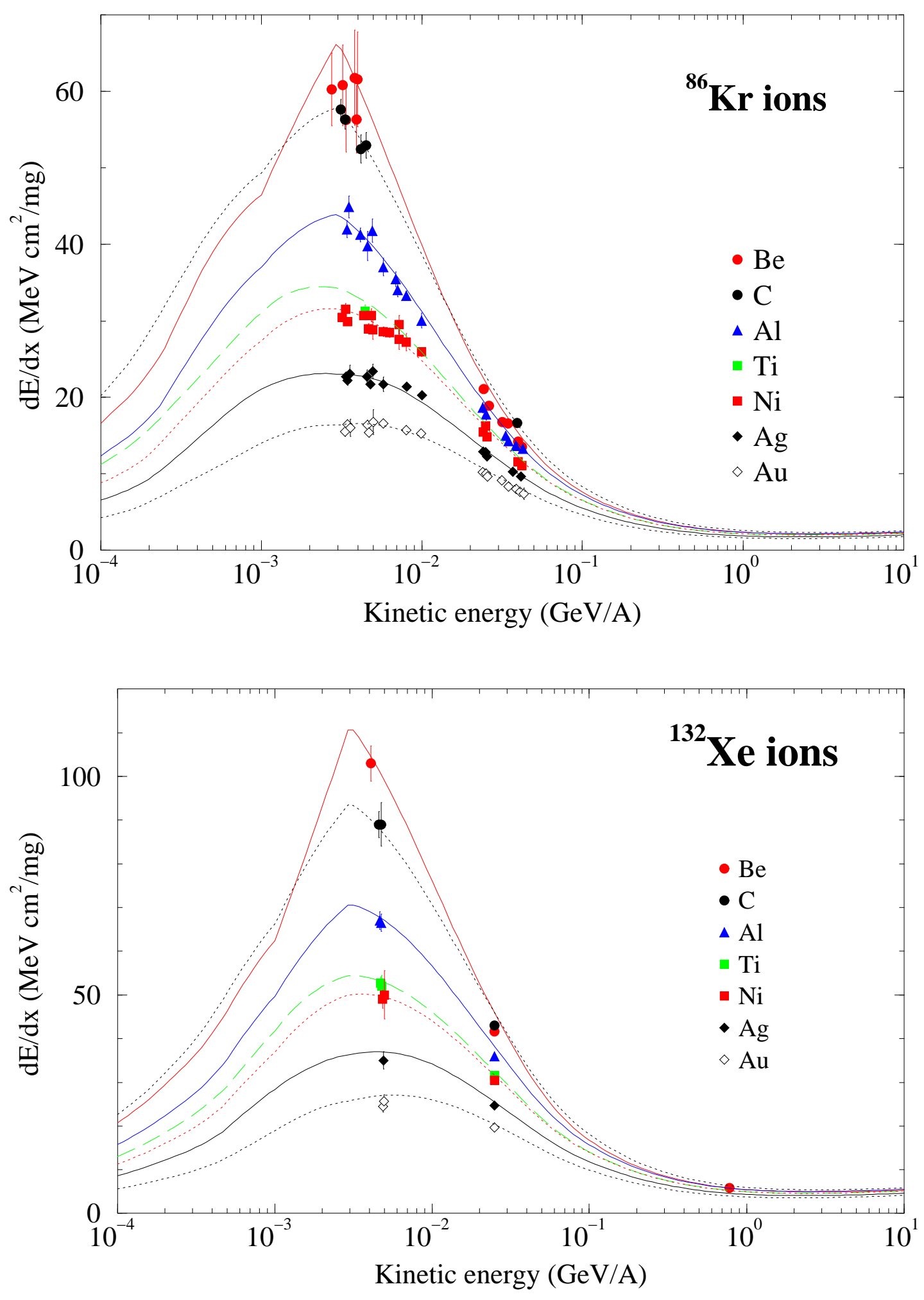

Figure 4: Comparisons between experimental data [5] (symbols) and our model (lines) on stopping power of ${ }^{86} \mathrm{Kr}$ and ${ }^{132} \mathrm{Xe}$ ions in various targets. 

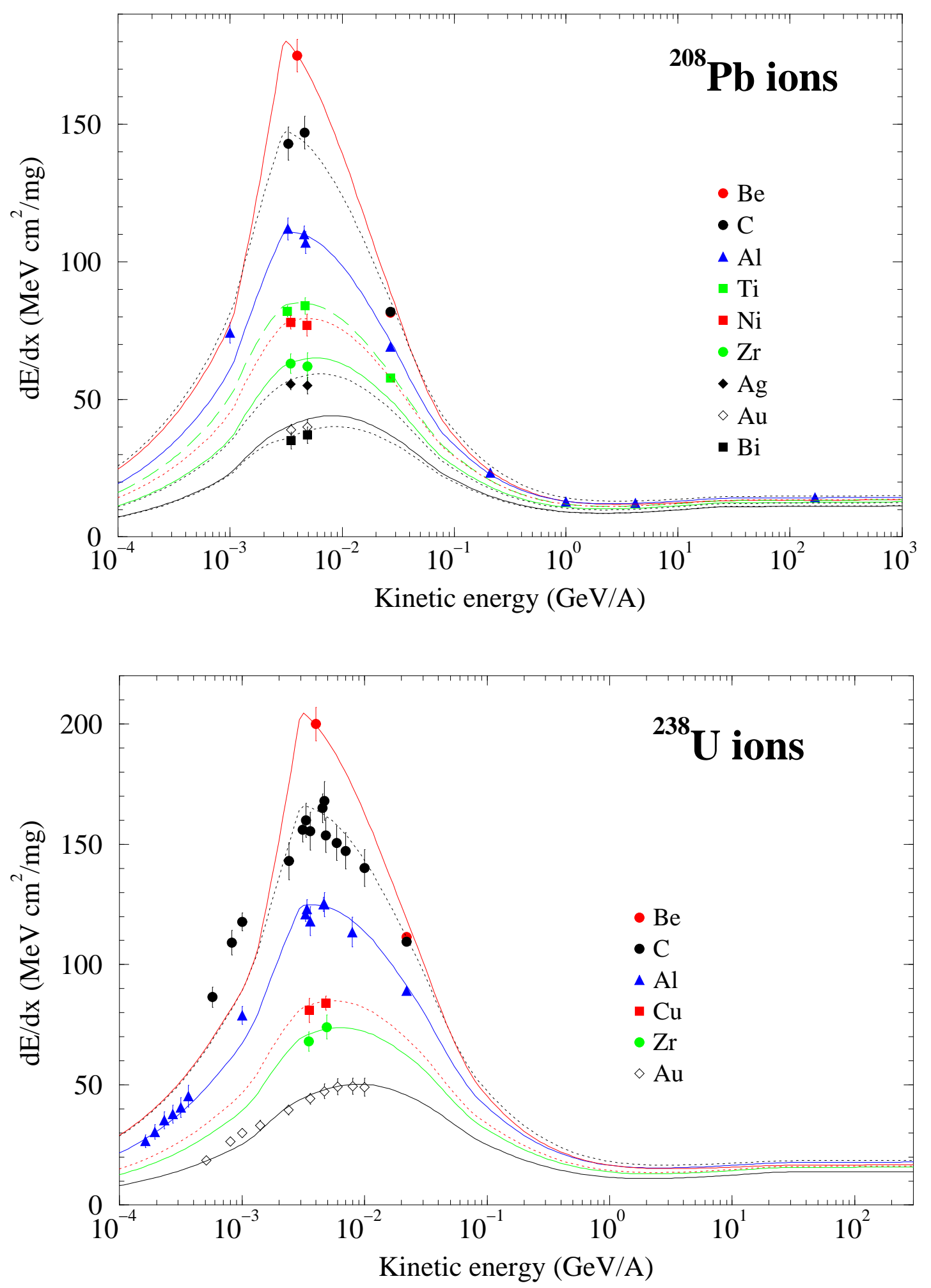

Figure 5: Comparisons between experimental data [5] (symbols) and our model (lines) on stopping power of ${ }^{208} \mathrm{~Pb}$ and ${ }^{238} \mathrm{U}$ ions in various targets. 


\subsection{Few-electron heavy ions}

Comparisons between experimental data and model predictions on stopping power of super heavy ions at energies between 100 and $1000 \mathrm{MeV} /$ nucleon with distributions of ion charge states taken into account according to the expression (3) are shown in Fig. 6.
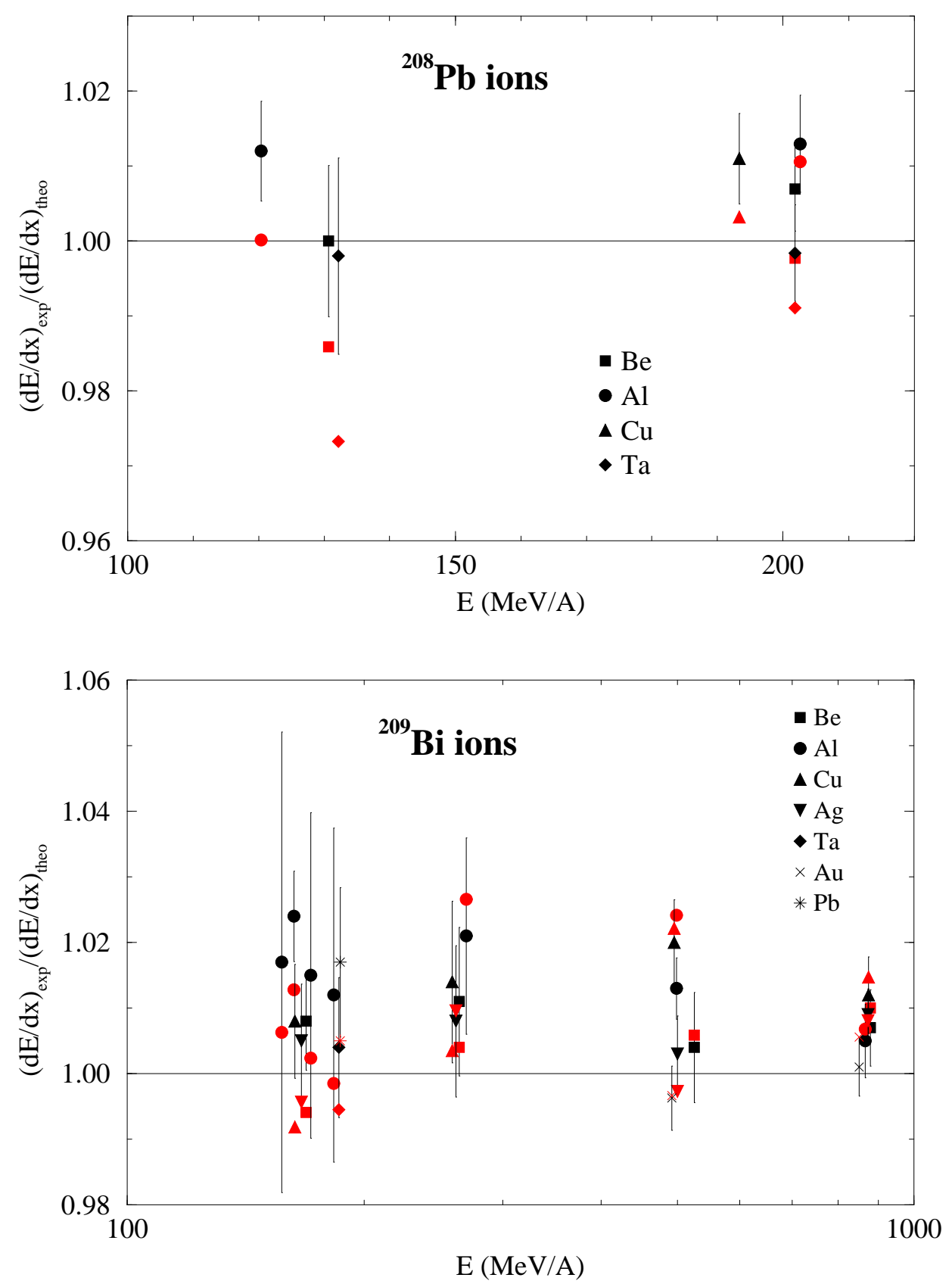

Figure 6: Comparisons between experimental data [4] and model predictions on stopping power of ${ }^{208} \mathrm{~Pb}$ and ${ }^{209} \mathrm{Bi}$ ions in various targets. Black symbols-code ATIMA [14], red symbols-present model. 
The overall agreement of our model with the experimental data looks good. The worst disagreement is observed for ${ }^{208} \mathrm{~Pb}$ ions on tantalum and it is about $3 \%$. The code ATIMA agrees with the experiment for such a projectile-target combination better. For all the other presented cases the code has no advantage over the presented model.

\section{Acknowledgment}

Author is grateful to Helmut Weick of GSI for helpful discussions and drawing attention to available information on distributions of ion charge states.

\section{References}

[1] N.V. Mokhov, “The MARS code system userś guide version 13(95)," Fermilab-FN628 (1995); N.V. Mokhov, S.I. Striganov, "MARS15 overview," Proc. Hadronic Shower Simulation Workshop, Batavia, Illinois, USA, 6-8 September, 2006, Vol. 896, pp. 50-60, American Institute of Physics, Melville, NY (2007); http://www-ap.fnal.gov/MARS/.

[2] I.L. Rakhno, N.V. Mokhov, S.I. Striganov, "Modeling Heavy Ion Ionization Loss in the MARS15 Code," Fermilab-Conf-05-019-AD (2005).

[3] T.E. Pierce and Marshall Blann, Phys. Rev., 173, pp. 390-405 (1968).

[4] H. Weick, H. Geissel, C. Scheidenberger et al., Nucl. Instrum. Meth. Phys. Res., B164-165, pp. 168-179 (2000).

[5] F. Hubert, R. Bimbot and H. Gauvin, Nucl. Instrum. Meth. Phys. Res., B36, pp. 357363 (1989).

[6] B.A. Weaver, A.J. Westphal, Nucl. Instrum. Meth. Phys. Res., B187, pp. 285-301 (2002).

[7] J.F. Ziegler, J.P.Biersack, M.D. Ziegler, "SRIM: The Stopping and Range of Ions in Matter," http://www.srim.org.

[8] H. Paul, A. Schinner, Nucl. Instrum. Meth. Phys. Res., B179, pp. 299-315 (2001); Ibid., B195, pp. 166-174 (2002).

[9] "Physical Reference Data," National Institute of Standards and Technology, http://physics.nist.gov/PhysRefData/contents.html.

[10] H. Bichsel, D.E. Groom, and S.R. Klein, Phys. Rev., D66, p. 010001-195 (2002).

[11] J. Lindhard and A. Sørensen, Phys. Rev., A53, p. 2443-2456 (1996).

[12] F. Hubert, R. Bimbot and H. Gauvin, Atomic Data and Nucl. Data Tables., 46, pp. $1-213$ (1990). 
[13] http://www-linux.gsi.de//weick/charge_states/.

[14] http://www-linux.gsi.de/ /weick/atima/atima.html. 